\title{
Aldosterone Blockade Reduces Mortality without Changing Cardiac Remodeling in Spontaneously Hypertensive Rats
}

\author{
Marcelo D.M. Cezara Ricardo L. Damatto ${ }^{a}$ Paula F. Martinez ${ }^{b} \quad$ Aline R.R. Lima $^{a}$ \\ Dijon H.S. Campos ${ }^{a}$ Camila M. Rosa ${ }^{a}$ Daniele M. Guizonia Camila Bonomo ${ }^{a}$ \\ Antonio C. Cicogna ${ }^{a}$ Rodrigo Gimenes ${ }^{a} \quad$ Luana U. Pagan ${ }^{a}$ Marina P. Okoshia \\ Katashi Okoshia
}

Department of Internal Medicine, Botucatu Medical School, Sao Paulo State University, UNESP, Botucatu

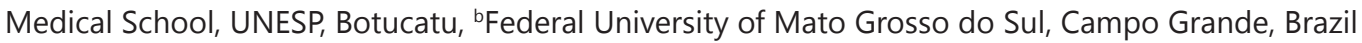

\section{Key Words}

Heart failure $\cdot$ Myocardial function Echocardiogram • Spironolactone $\cdot$ Ventricular function - Papillary muscle

\begin{abstract}
Background: The role of aldosterone blockers during transition from long-term compensated hypertrophy to dilated failure is not completely understood. In this study we evaluated the effects of early administration of spironolactone on cardiac remodeling, myocardial function, and mortality in spontaneously hypertensive rats (SHR). Methods: Sixteen-month-old SHR received no treatment (SHR-C, $n=72$ ) or spironolactone (SHR-SPR, $20 \mathrm{mg} / \mathrm{kg} / \mathrm{day}, \mathrm{n}=34$ ) for six months. Echocardiogram was performed before and after treatment. Myocardial function was analyzed in left ventricular (LV) papillary muscle preparations. Myocardial collagen and hydroxyproline concentration were evaluated by morphometry and spectrophotometry, respectively. LV gene expression was assessed by real time RT-PCR. Statistics: Student's t test; Log rank test (Kaplan Meyer). Results: SHR-C and SHR-SPR presented mortality rates of 71 and $38 \%$, respectively $(p=0.004)$. Systolic arterial pressure did not differ between groups (SHR-C 199 \pm 43 ; SHR-SPR $200 \pm 35 \mathrm{mmHg}$ ). Initial and final echocardiograms did not show significant differences in cardiac structures or LV function between groups. Myocardial function was similar between groups at basal and after inotropic stimulation. Collagen fractional area, hydroxyproline concentration, gene expression for $\alpha$ - and $\beta$-myosin heavy chain, atrial natriuretic peptide, and Serca2a were not different between groups. Conclusion: Early spironolactone administration reduces mortality without changing cardiac remodeling in spontaneous hypertensive rats.
\end{abstract}

Copyright $@ 2013$ S. Karger AG, Basel 


\section{Introduction}

Chronic pressure overload is a major cause of heart failure. During sustained pressure overload, stable cardiac hypertrophy develops and may progress to a decompensated state with left ventricular dilation and systolic pump failure [1,2]. The mineralocorticoid hormone aldosterone has been shown to be involved in the pathophysiology of heart failure $[3,4]$. However, the role of aldosterone blockers during transition from long-term compensated hypertrophy to dilated failure is not completely understood.

Experimental models have shown that aldosterone induces deleterious cardiovascular effects such as myocardial fibrosis, myocyte hypertrophy and apoptosis, oxidative stress, vascular injury, endothelial dysfunction, renal retention of sodium and water, loss of potassium and magnesium, electrical remodeling, and sudden death [5-11]. Two aldosterone blockers, spironolactone and eplerenone have been evaluated in clinical and experimental studies [12-24]. In different models of cardiac injury, aldosterone blockers can prevent or attenuate left ventricular structural, functional, and molecular changes [12-20]. Two large clinical trials have shown that aldosterone receptor blockers reduce mortality and hospitalizations in patients with systolic heart failure of any cause and New York Heart Association functional classes III and IV (RALES) [21], and in patients with systolic dysfunction post-myocardial infarction (EPHESUS) [22]. The EMPHASIS-HF study showed that aldosterone blocker eplerenone reduced mortality and hospitalizations in patients with heart failure of any cause in functional class II and left ventricular ejection fraction $\leq 0.35$ [23]. Despite being now recommended for symptomatic systolic heart failure patients [25], evidence is less consistent on the benefits of the aldosterone blockers, in milder forms of heart failure.

The spontaneously hypertensive rat (SHR) is a widely used experimental model for studying heart failure $[1,26,27]$. It presents early arterial hypertension and left ventricular hypertrophy which evolves to heart failure during maturity and senescence. As cardiac remodeling and heart failure development is slow, SHRs are considered a useful model to mimic clinical heart failure settings. In this study we evaluated the effects of chronic administration of aldosterone blocker spironolactone, introduced before the appearance of heart failure clinical signs, on cardiac remodeling, myocardial function, and mortality in spontaneously hypertensive rats.

\section{Materials and Methods}

\section{Experimental groups}

Male spontaneously hypertensive rats (SHR) were purchased from the Central Animal House at Botucatu Medical School, UNESP. All animals were housed in a room under temperature control at $23{ }^{\circ} \mathrm{C}$ and kept on a 12-hour light/dark cycle. Food and water were supplied ad libitum. All experiments and procedures were approved by the Ethics Committee of Botucatu Medical School, UNESP, Botucatu, SP, Brazil.

Sixteen-month-old SHR were divided into two groups: control (SHR-C, n=72) and spironolactone (SHRSPR, $n=34$ ). Spironolactone (Biolab Pharmaceutical, Sao Paulo, Brazil) was added to rat chow at $20 \mathrm{mg} / \mathrm{kg} /$ day for six months. Rats were weighed once weekly to adjust for drug dosage. Systolic arterial pressure was measured by tail-cuff method at 16 and 22 months old. During euthanasia, we assessed pathological features of congestive heart failure. Rats with pleuropericardial effusion, left atrial thrombi, and/or right ventricular hypertrophy (right ventricle weight-to-body weight ratio $>0.8 \mathrm{mg} / \mathrm{g}$ ) were considered to present heart failure $[1,2,26,28]$.

\section{Echocardiographic study}

Echocardiographic evaluation was performed using a commercially available echocardiograph (General Electric Medical Systems, Vivid S6, Tirat Carmel, Israel) equipped with a 5 - $11.5 \mathrm{MHz}$ multifrequency probe. Rats were anesthetized by intramuscular injection of a mixture of ketamine $(50 \mathrm{mg} / \mathrm{kg})$ and xylazine $(0.5$ $\mathrm{mg} / \mathrm{kg}$ ). A two-dimensional parasternal short-axis view of the left ventricle (LV) was obtained at the level of the papillary muscles. M-mode tracings were obtained from short-axis views of the LV at or just below the tip of the mitral-valve leaflets, and at the level of the aortic valve and left atrium [29-32]. M-mode images of 
the LV were printed on a black-and-white thermal printer (Sony UP-890MD) at a sweep speed of $100 \mathrm{~mm} / \mathrm{s}$. All LV structures were manually measured by the same observer according to the leading-edge method of the American Society of Echocardiography [33]. The measurements obtained were the mean of at least five cardiac cycles on the M-mode tracings. The following structural variables were measured: left atrium (LA) diameter, LV diastolic and systolic dimensions (LVDD and LVSD, respectively), LV diastolic posterior wall thickness (PWT), LV diastolic septal wall thickness (SWT), and aortic diameter (AO). Left ventricular weight $(\mathrm{LVW})$ was calculated using the formula [(LVDD + PWT + SWT $\left.)^{3}-(\mathrm{LVDD})^{3}\right]$ x 1,04 [34]. Left ventricular function was assessed by the following parameters: endocardial fractional shortening (EFS), midwall fractional shortening (MWFS), ejection fraction (EF), posterior wall shortening velocity (PWSV), early and late diastolic mitral inflow velocities ( $\mathrm{E}$ and $\mathrm{A}$ waves), E/A ratio, and isovolumetric relaxation time (IVRT).

\section{Myocardial functional study}

At the end of the experimental period, two days after the echocardiographic study, myocardial intrinsic contractile performance was evaluated in isolated LV papillary muscle preparation as previously described [35-37]. The rats were anesthetized (pentobarbital sodium, $50 \mathrm{mg} / \mathrm{kg}$, intraperitoneally) and decapitated. Hearts were quickly removed and placed in oxygenated Krebs-Henseleit solution at $28^{\circ} \mathrm{C}$. Left ventricular anterior or posterior papillary muscle was dissected free, mounted between two spring clips, and placed vertically in a chamber containing Krebs-Henseleit solution at $28^{\circ} \mathrm{C}$ and oxygenated with a mixture of 95 $\% \mathrm{O}_{2}$ and $5 \% \mathrm{CO}_{2}(\mathrm{pH} 7.38$ ). The composition of the Krebs-Henseleit solution in mM was as follows: 118.5 $\mathrm{NaCl}, 4.69 \mathrm{KCl}, 1.25 \mathrm{CaCl}_{2}, 1.16 \mathrm{MgSO}_{4}, 1.18 \mathrm{KH}_{2} \mathrm{PO}_{4}, 5.50$ glucose, and $25.88 \mathrm{NaHCO}_{3}$. The spring clips were attached to a Kyowa model 120T-20B force transducer and a lever system, which allowed for muscle length adjustment. Preparations were stimulated 12 times/min at a voltage $10 \%$ above threshold.

After a 60-min period, during which the preparations were permitted to shorten while carrying light loads, muscles were loaded to contract isometrically and stretched to the apices of their length-tension curves $\left(\mathrm{L}_{\max }\right)$. After a 5-min period, during which preparations performed isotonic contractions, muscles were again placed under isometric conditions, and the apex of the length-tension curve was determined. A 15-min period of stable isometric contraction was imposed prior to the experimental period. One isometric contraction was then recorded for later analysis.

The following parameters were measured from isometric contraction: peak of developed tension (DT, g/ $\mathrm{mm}^{2}$ ), resting tension (RT, $\mathrm{g} / \mathrm{mm}^{2}$ ), time to peak of tension (TPT, ms), maximum rate of tension development $\left(+\mathrm{dT} / \mathrm{dt}, \mathrm{g} / \mathrm{mm}^{2} / \mathrm{s}\right)$, and maximum rate of tension decline $\left(-\mathrm{dT} / \mathrm{dt}, \mathrm{g} / \mathrm{mm}^{2} / \mathrm{s}\right)$. To evaluate contractile reserve and myocardial response to $\beta$-adrenergic stimulation, mechanical performance of papillary muscles were evaluated in basal condition and after the following inotropic stimulation: post-rest contraction, extracellular $\mathrm{Ca}^{2+}$ concentration increase, and beta-adrenergic agonist isoproterenol addition to the nutrient solution.

Papillary muscle cross-sectional area was calculated from muscle weight and length by assuming cylindrical uniformity and a specific gravity of 1.0. All force data were normalized for the muscle cross-sectional area.

After dissecting papillary muscle, lungs, atria, and ventricles were separated and weighed. Fragments of ventricles, atria, lung, and liver were weighed before and after drying sessions $\left(65^{\circ} \mathrm{C}\right.$ for $\left.72 \mathrm{~h}\right)$ to evaluate the wetto-dry weight ratio. Tibia was removed and dried and the lengths measured. Atria and left and right ventricular wet weight normalized by body weight and tibia length were used as indices of ventricular hypertrophy.

\section{Morphologic study}

Transverse LV sections were fixed in $10 \%$ buffered formalin and embedded in paraffin. Five-micrometerthick sections were stained with hematoxylin-eosin and the collagen-specific stain picrosirius red (Sirius red F3BA in aqueous saturated picric acid) [38]. At least 150 fiber cross-sectional areas were measured from each heart. On average, 20 microscopic fields were used to quantify interstitial collagen fractional area. Perivascular collagen was excluded from this analysis. Measurements were performed using a Leica microscope (magnification 40X) attached to a video camera and connected to a computer equipped with image analysis software (Image-Pro Plus 3.0, Media Cybernetics, Silver Spring, MD, USA).

\section{Myocardial hydroxyproline and serum electrolytes concentration}

Myocardial hydroxyproline (HOP) concentration was assessed for tissue collagen content estimation. HOP was measured in LV tissue as previously described [39]. Briefly, the tissue was dried using a Speedvac 
Concentrator SC 100 attached to a refrigerated condensation trap (TRL 100) and vacuum pump (VP 100, Savant Instruments, Inc., Farmingdale, NY, USA). Tissue dry weight was measured and the samples were hydrolyzed overnight at $100{ }^{\circ} \mathrm{C}$ with $6 \mathrm{~N} \mathrm{HCl}(1 \mathrm{ml} / 10 \mathrm{mg}$ dry tissue $)$. A $50 \mu \mathrm{l}$ aliquot of the hydrolysate was transferred to an Eppendorf tube and dried in the Speedvac Concentrator. One milliliter of deionized water was added and the sample transferred to a tube with a Teflon screw cap. One milliliter of potassium borate buffer $(\mathrm{pH}$ 8.7) was added to maintain constant $\mathrm{pH}$ and the sample was oxidized with $0.3 \mathrm{ml}$ of chloramine $\mathrm{T}$ solution at room temperature for $20 \mathrm{~min}$. The addition of $1 \mathrm{ml}$ of $3.6 \mathrm{~mol} / \mathrm{l}$ sodium thiosulfate and thorough mixing for 10 $\mathrm{s}$ stopped the oxidative process. The solution was saturated with $1.5 \mathrm{~g} \mathrm{KCl}$. The tubes were capped and heated in boiling water for $20 \mathrm{~min}$. After cooling to room temperature, the aqueous layer was extracted with $2.5 \mathrm{ml}$ of toluene. One and a half milliliters of toluene extract were transferred to a $12 \mathrm{X} 75 \mathrm{~mm}$ test tube. Then $0.6 \mathrm{ml}$ of Ehrlich's reagent was added and the color allowed to develop for $30 \mathrm{~min}$. Absorbances were read at $565 \mathrm{~mm}$ against a reagent blank. Deionized water and $20 \mu \mathrm{g} / \mathrm{ml} \mathrm{HOP}$ were used as the blank and standard, respectively.

Sodium, potassium, and magnesium serum concentration was analyzed by flame photometry (FC-280, CELM) using a commercial standard solution.

\section{Gene expression}

To evaluate expression of the fetal gene program, we assessed $\alpha$ - and $\beta$-myosin heavy chain, atrial natriuretic peptide (ANP), and sarcoplasmic reticulum calcium ATPase (Serca 2a) gene expression by real time RT-PCR (transcription polymerase chain reaction after reverse transcription). Total RNA was extracted from LV myocardial sample with TRIzol Reagent (Invitrogen Life Technologies, Carlsbad, CA, USA) as previously described [40-42]. Frozen muscles were mechanically homogenized on ice in $1 \mathrm{ml}$ of ice-cold TRIzol reagent. Total RNA was solubilized in RNase-free $\mathrm{H}_{2} \mathrm{O}$, incubated in DNase I (Invitrogen Life Technologies) to remove any DNA in the sample, and quantified by measuring optical density (OD) at $260 \mathrm{~nm}$. RNA purity was ensured by obtaining a $260 / 280 \mathrm{~nm}$ OD ratio of approximately 2.0. One $\mu \mathrm{g}$ of RNA was reverse transcribed using High Capacity cDNA Reverse Transcription Kit in a total volume of $20 \mu \mathrm{l}$, according to standard methods (Applied Biosystems, Foster City, CA, EUA). Aliquots of $2.5 \mu \mathrm{l}$ (10-100 ng) cDNA were then submitted to real-time PCR reaction using $10 \mu \mathrm{l} 2 \mathrm{X}$ TaqMan $囚$ Universal PCR Master Mix (Applied Biosystems) and $1 \mu \mathrm{l}$ of customized assay (20X) containing sense and antisense primers and Taqman (Applied Biosystems, Foster City, CA, EUA) probe specific to each gene, alpha myosin (myosin heavy polypeptide 6, cardiac muscle, alpha; Taqman assay Rn00568304_m1; Ref. seq. Genbank NM_017239.1), beta myosin (myosin heavy polypeptide 7, cardiac muscle, beta; Taqman assay Rn00568328_m1; Ref. seq. Genbank NM_017240.1), natriuretic peptide precursor type A (Taqman assay Rn00561661_m1; Ref. seq. Genbank NM_012612.1), and Serca2a (Taqman assay Rn00568762; Ref. seq. Genbank NM_017290). Amplification and analysis were performed using a StepOnePlus ${ }^{\mathrm{TM}}$ Real Time PCR System (Applied Biosystems, Foster City, CA, EUA) according to manufacturer's recommendation. Expression data were normalized to cyclophilin expression (reference gene; Taqman assay Rn00690933_m1; Ref. seq. Genbank NM_017101). Reactions were performed in triplicate and expression levels calculated using the CT comparative method $\left(2^{-\Delta \Delta C T}\right)$.

\section{Western blotting analysis}

To evaluate expression of the proteins involved in intracellular calcium transient, we performed Western blot analyses in LV samples according to a previously described method [43] with specific anti-Serca 2 ATPase (MA3-910, IID8) and anti-phospholamban (MA3-922, 2D12) antibodies (Thermo Fisher Scientific, Rockford, IL). Protein levels were normalized to those of GAPDH (6C5, sc-32233, Santa Cruz Biotechnology). Muscle protein was extracted using Tris-Triton buffer (10 mM Tris pH 7.4, $100 \mathrm{mM} \mathrm{NaCl}, 1 \mathrm{mM}$ EDTA, 1 mM EGTA, 1\% Triton X-100, $10 \%$ glycerol, $0.1 \%$ SDS, $0.5 \%$ deoxycholate). Supernatant protein content was quantified by the Bradford method. Samples were separated on a polyacrylamide gel and then transferred to a nitrocellulose membrane. After blockade, the membrane was incubated with the primary antibody and then washed with TBS and Tween 20 and incubated with secondary peroxidase-conjugated antibody. Super Signal $₫$ West Pico Chemiluminescent Substrate (Pierce Protein Research Products, Rockford, USA) was used to detect bound antibodies.

\section{Statistical analysis}

Data are expressed as mean \pm standard deviation or median and $25^{\text {th }}$ and $75^{\text {th }}$ percentiles. Comparisons between the groups were performed by Student's $t$ test or Mann-Whitney test. Mortality was assessed by 


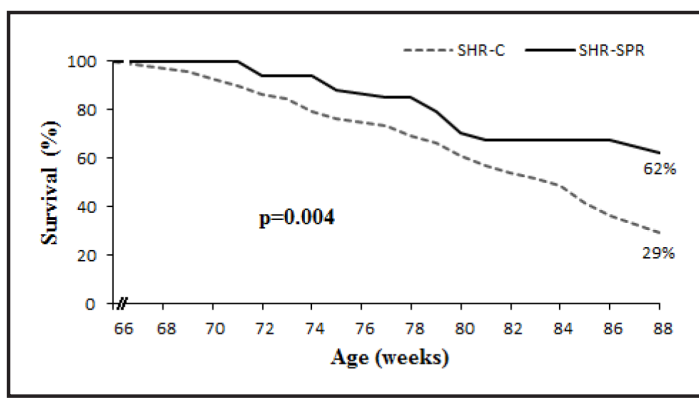

Fig. 1. Probability of survival; Log rank test: Kaplan-Meier. SHR-C: spontaneously hypertensive rats without treatment; SHR-SPR: spontaneously hypertensive rats treated with spironolactone.

\begin{tabular}{lccc}
\hline Variables & $\begin{array}{c}\text { SHR-C } \\
(\mathrm{n}=21)\end{array}$ & $\begin{array}{c}\text { SHR-SPR } \\
(\mathrm{n}=21)\end{array}$ & P value \\
\hline Initial BW (g) & $393 \pm 23$ & $393 \pm 27$ & 0.985 \\
Final BW (g) & $347 \pm 34^{*}$ & $348 \pm 33^{*}$ & 0.975 \\
Initial SBP (mmHg) & $206 \pm 17$ & $215 \pm 21$ & 0.259 \\
Final SBP (mmHg) & $199 \pm 43$ & $200 \pm 35$ & 0.932 \\
\hline
\end{tabular}

Table 1. Body weight and systolic blood pressure. Data are expressed as mean \pm standard deviation. SHR-C: spontaneously hypertensive rats without treatment; SHR-SPR: spontaneously hypertensive rats treated with spironolactone; BW: body weight; SBP: systolic blood pressure. ${ }^{*} \mathrm{p}<0.05$ vs. Initial BW (paired Student's $t$ test); P value: SHR-SPR vs. SHR-C (unpaired Student's $t$ test)

\begin{tabular}{lccc}
\hline Variables & $\begin{array}{c}\text { SHR-C } \\
(\mathrm{n}=11)\end{array}$ & $\begin{array}{c}\text { SHR-SPR } \\
(\mathrm{n}=19)\end{array}$ & P value \\
\hline HR (bpm) & $329 \pm 29$ & $334 \pm 28$ & 0.665 \\
LVDD (mm) & $8.87 \pm 1.26$ & $8.66 \pm 1.13$ & 0.641 \\
LVSD (mm) & $4.85 \pm 1.58$ & $4.96 \pm 1.35$ & 0.849 \\
LVPWT (mm) & $1.93 \pm 0.14$ & $1.82 \pm 0.15$ & 0.059 \\
LVSWT (mm) & $1.94 \pm 0.13$ & $1.86 \pm 0.15$ & 0.189 \\
AO (mm) & $4.97 \pm 0.34$ & $4.78 \pm 0.40$ & 0.199 \\
LA (mm) & $7.52 \pm 1.42$ & $7.65 \pm 1.30$ & 0.802 \\
LV weight (g) & $1.45 \pm 0.39$ & $1.31 \pm 0.36$ & 0.338 \\
RWT & $0.44 \pm 0.07$ & $0.42 \pm 0.06$ & 0.473 \\
\hline
\end{tabular}

\begin{tabular}{lccc}
\hline Variables & $\begin{array}{c}\text { SHR-C } \\
(\mathrm{n}=11)\end{array}$ & $\begin{array}{c}\text { SHR-SPR } \\
(\mathrm{n}=19)\end{array}$ & P value \\
\hline EFS $(\%)$ & $46.4 \pm 10.9$ & $43.7 \pm 8.81$ & 0.453 \\
MWFS $(\%)$ & $26.8 \pm 5.65$ & $25.2 \pm 4.93$ & 0.426 \\
EF & $0.83 \pm 0.10$ & $0.81 \pm 0.09$ & 0.575 \\
PWSV (mm/s) & $33.2 \pm 6.73$ & $29.7 \pm 4.78$ & 0.115 \\
E-wave (cm/s) & $57.0(47.5-86.8)$ & $70.0(52.0-125)$ & 0.464 \\
A-wave (cm/s) & $82.4 \pm 35.7$ & $69.0 \pm 34.3$ & 0.320 \\
E/A & $0.63(0.54-0.74)$ & $0.71(0.59-3.65)$ & 0.606 \\
IVRT (ms) & $36.5 \pm 7.08$ & $38.7 \pm 4.68$ & 0.327 \\
\hline
\end{tabular}

Table 3. Echocardiographic left ventricular func-

Table 2. Echocardiographic cardiac structural pa- tional parameters. Data are expressed as mean \pm rameters. Data are expressed as mean \pm standard standard deviation or median and $25^{\text {th }}$ and $75^{\text {th }}$ deviation. SHR-C: spontaneously hypertensive rats percentiles. SHR-C: spontaneously hypertensive without treatment; SHR-SPR: spontaneously hyper- rats without treatment; SHR-SPR: spontaneously tensive rats treated with spironolactone. HR: heart hypertensive rats treated with spironolactone. EFS: rate; LVDD and LVSD: left ventricular (LV) diastolic endocardial fractional shortening; MWFS: midwall and systolic diameter, respectively; LVPWT: LV poste- fractional shortening; EF: ejection fraction; PWSV: rior wall thickness; LVSWT: LV septal wall thickness; posterior wall shortening velocity; E-wave and AAO: aorta diameter; LA: left atrial diameter; RWT: wave: early and late diastolic mitral inflow, respecrelative wall thickness. Unpaired Student's $t$ test tively; IVRT: isovolumetric relaxation time. Unpaired Student's $t$ test

log-rank test (Kaplan Meier). Analysis of papillary muscle studies using different inotropic stimulation was performed by repeated measures ANOVA. Statistical significance was accepted at the level of $p<0.05$.

\section{Results}

During the experimental period, SHR-C and SHR-SPR presented mortality rates of $71 \%$ and $38 \%$, respectively ( $\mathrm{p}=0.004$ ). Survival curves according to animal age are shown in Figure 1. In surviving animals ( $\mathrm{n}=21$ per group) no difference was seen in the percentage of pathologically diagnosed heart failure (76.2\%) between groups.

Initial and final body weight was not statistically different between groups; both groups presented a significant reduction in final body weight compared to initial weight. Initial and final systolic blood pressure was not different between groups (Table 1). Transthoracic echocardiography was performed at the beginning and end of the experiment. The initial exam aimed to ensure homogeneity between groups (data not shown). In the final exam, no significant differences were observed in cardiac structural and functional parameters (Tables 2 and 3). 


\begin{tabular}{lccc}
\hline Variables & $\begin{array}{c}\text { SHR-C } \\
(\mathrm{n}=18)\end{array}$ & $\begin{array}{c}\text { SHR-SPR } \\
(\mathrm{n}=20)\end{array}$ & P value \\
\hline DT $\left(\mathrm{g} / \mathrm{mm}^{2}\right)$ & $4.49 \pm 1.79$ & $4.70 \pm 1.67$ & 0.706 \\
RT $\left(\mathrm{g} / \mathrm{mm}^{2}\right)$ & $0.88 \pm 0.24$ & $0.89 \pm 0.28$ & 0.918 \\
TPT $(\mathrm{ms})$ & $221 \pm 16$ & $218 \pm 17$ & 0.611 \\
$+\mathrm{dT} / \mathrm{dt}\left(\mathrm{g} / \mathrm{mm}^{2} / \mathrm{s}\right)$ & $38.0 \pm 17.9$ & $39.1 \pm 15.7$ & 0.838 \\
$-\mathrm{dT} / \mathrm{dt}\left(\mathrm{g} / \mathrm{mm}^{2} / \mathrm{s}\right)$ & $19.0 \pm 6.28$ & $19.5 \pm 5.66$ & 0.779 \\
PM CSA $\left(\mathrm{mm}^{2}\right)$ & $1.19 \pm 0.38$ & $1.15 \pm 0.27$ & 0.699 \\
\hline
\end{tabular}

Table 4. Basal data of isolated papillary muscle. Data are expressed as mean \pm standard deviation. SHR-C: spontaneously hypertensive rats without treatment; SHR-SPR: spontaneously hypertensive rats treated with spironolactone. DT: peak of developed tension; RT: resting tension; TPT: time to peak of tension; $+\mathrm{dT} / \mathrm{dt}$ : maximum rate of tension development; -dT/ $\mathrm{dt}$ : maximum rate of tension decline; PM CSA: papillary muscle cross sectional area. Unpaired Student's $t$ test

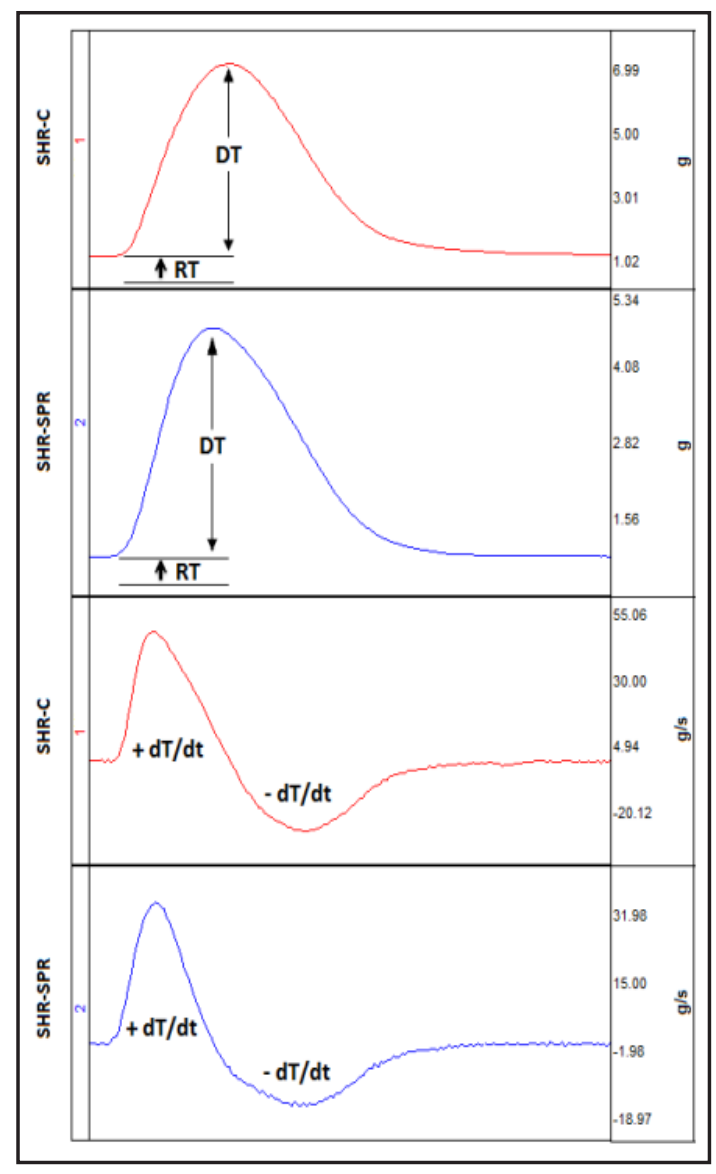

Fig. 2. Illustrative recordings of papillary muscles during isometric contractions at extracellular calcium concentration of $1.25 \mathrm{mM}$. SHR-C: spontaneously hypertensive rats without treatment; SHRSPR: spontaneously hypertensive rats treated with spironolactone; DT: peak of developed tension; RT: resting tension; $+\mathrm{dT} / \mathrm{dt}$ : maximum rate of tension development; -dT/dt: maximum rate of tension decline.

Papillary muscle functional data at basal conditions with an extracellular calcium concentration of $1.25 \mathrm{mM}$ were not different between groups (Table 4). Figure 2 shows representative recordings of isometric contractions for both SHR-C and SHR-SPR groups. All parameters obtained at post-rest contractions and after extracellular $\mathrm{Ca}^{2+}$ concentration increase were not different between groups (data not shown). Table 5 shows papillary muscle data under isometric contraction after stimulation with different doses of $\beta$-adrenergic agonist isoproterenol. There were no differences between groups for all parameters.

Anatomical parameters LV, right ventricle, atria, and lung weights and their wet/dry ratio were not statistically different between groups. Also, LV, right ventricle, and atria weights normalized to body weight or tibia length did not differ between groups (Table 6). Myocyte cross sectional area was lower in the SHR-SPR than SHR-C. Myocardial interstitial collagen fractional area and hydroxyproline concentration were not statistically different between groups (Table 7). Serum electrolytes sodium (SHR-C $145 \pm 4.38$; SHR-SPR $141 \pm 3.62 \mathrm{mEq} / \mathrm{L}$ ), potassium (SHR-C $4.31 \pm 0.36$; SHR-SPR $4.46 \pm 0.42 \mathrm{mEq} / \mathrm{L}$ ), and magnesium (SHR-C $2.11 \pm 0.21$; SHR-SPR 


\begin{tabular}{|c|c|c|}
\hline Cellular Phy & Cell Physiol Biochem 2013;32:1275-1287 & \\
\hline and Biochemistry & $\begin{array}{l}\text { DOI: 10.1159/000354526 } \\
\text { Published online: November 22, } 2013\end{array}$ & $\begin{array}{l}\text { (c) } 2013 \text { S. Karger AG, Basel } \\
\text { www.karger.com/cpb }\end{array}$ \\
\hline
\end{tabular}

\begin{tabular}{lcccccc}
\hline & $\mathrm{mM}$ & $\mathrm{DT}\left(\mathrm{g} / \mathrm{mm}^{2}\right)$ & $\mathrm{RT}\left(\mathrm{g} / \mathrm{mm}^{2}\right)$ & $\mathrm{TPT}(\mathrm{ms})$ & $+\mathrm{dT} / \mathrm{dt}\left(\mathrm{g} / \mathrm{mm}^{2} / \mathrm{s}\right)$ & $-\mathrm{dT} / \mathrm{dt}\left(\mathrm{g} / \mathrm{mm}^{2} / \mathrm{s}\right)$ \\
\hline SHR-C & ISO $10^{-8}$ & $4.30 \pm 1.76$ & $0.75 \pm 0.24$ & $190 \pm 12$ & $40.0 \pm 16.9$ & $19.0 \pm 6.15$ \\
$(\mathrm{n}=18)$ & ISO $10^{-7}$ & $4.09 \pm 1.85$ & $0.74 \pm 0.24$ & $162 \pm 14$ & $43.5 \pm 20.8$ & $23.0 \pm 6.49$ \\
& ISO $10^{-6}$ & $3.92 \pm 1.82$ & $0.72 \pm 0.24$ & $156 \pm 8$ & $44.7 \pm 22.9$ & $27.6 \pm 10.3$ \\
SHR-SPR & ISO $10^{-8}$ & $4.32 \pm 1.62$ & $0.76 \pm 0.27$ & $200 \pm 18$ & $41.8 \pm 17.5$ & $19.8 \pm 6.78$ \\
$(\mathrm{n}=20)$ & ISO $10^{-7}$ & $3.91 \pm 1.69$ & $0.74 \pm 0.26$ & $168 \pm 10$ & $43.1 \pm 19.1$ & $22.6 \pm 9.60$ \\
& ISO $10^{-6}$ & $3.75 \pm 1.66$ & $0.73 \pm 0.28$ & $154 \pm 13$ & $44.4 \pm 20.3$ & $26.4 \pm 11.1$ \\
\hline
\end{tabular}

Table 5. Papillary muscle data under isometric contraction after stimulation with the $\beta$-adrenergic agonist isoproterenol. Data are expressed as mean \pm standard deviation. SHR-C: spontaneously hypertensive rats without treatment; SHR-SPR: spontaneously hypertensive rats treated with spironolactone. ISO: isoproterenol; DT: peak of developed tension; RT: resting tension; TPT: time to peak of tension; $+\mathrm{dT} / \mathrm{dt}$ : maximum rate of tension development; -dT/dt: maximum rate of tension decline. Repeated measures ANOVA

Table 6. Anatomical data. Data are expressed as mean \pm standard deviation. SHR-C: spontaneously hypertensive rats without treatment; SHR-SPR: spontaneously hypertensive rats treated with spironolactone. LVW: left ventricle weight; BW: body weight; RVW: right ventricle weight; wet/dry: wet weight-to-dry weight ratio. Unpaired Student's $t$ test

\begin{tabular}{lccc}
\hline Variables & $\begin{array}{c}\text { SHR-C } \\
(\mathrm{n}=21)\end{array}$ & $\begin{array}{c}\text { SHR-SPR } \\
(\mathrm{n}=21)\end{array}$ & P value \\
\hline Tibia length (cm) & $4.28 \pm 0.08$ & $4.27 \pm 0.09$ & 0.714 \\
LVW (g) & $1.19 \pm 0.19$ & $1.22 \pm 0.20$ & 0.654 \\
LVW/BW (g/kg) & $3.41 \pm 0.42$ & $3.51 \pm 0.57$ & 0.552 \\
LVW/tibia (mg/cm) & $278 \pm 46$ & $285 \pm 47$ & 0.628 \\
RVW (g) & $0.36 \pm 0.09$ & $0.37 \pm 0.10$ & 0.808 \\
RVW/BW (g/kg) & $1.06 \pm 0.27$ & $1.07 \pm 0.28$ & 0.848 \\
RVW/tibia (mg/cm) & $85.1 \pm 21.1$ & $87.0 \pm 23.2$ & 0.780 \\
Atria (g) & $0.20 \pm 0.06$ & $0.20 \pm 0.05$ & 0.966 \\
Atria/BW (g/kg) & $0.59 \pm 0.18$ & $0.59 \pm 0.15$ & 0.972 \\
Atria/tibia (mg/cm) & $47.7 \pm 15.0$ & $47.6 \pm 11.8$ & 0.992 \\
LV wet/dry & $4.26 \pm 0.28$ & $4.30 \pm 0.18$ & 0.601 \\
RW wet/dry & $4.24 \pm 0.60$ & $4.27 \pm 0.54$ & 0.868 \\
Atria wet/dry & $4.66 \pm 0.26$ & $4.81 \pm 0.26$ & 0.069 \\
Lung (g) & $3.81 \pm 0.70$ & $3.71 \pm 0.81$ & 0.666 \\
Lung wet/dry & $5.21 \pm 0.42$ & $5.32 \pm 0.47$ & 0.458 \\
Liver wet/dry & $3.33 \pm 0.30$ & $3.27 \pm 0.28$ & 0.470 \\
\hline
\end{tabular}

\begin{tabular}{lccc}
\hline Variables & $\begin{array}{c}\text { SHR-C } \\
(\mathrm{n}=8)\end{array}$ & $\begin{array}{c}\text { SHR-SPR } \\
(\mathrm{n}=8)\end{array}$ & P value \\
\hline CSA $\left(\mu \mathrm{m}^{2}\right)$ & $550 \pm 34$ & $440 \pm 51$ & 0.001 \\
ICF $(\%)$ & $8.20 \pm 3.00$ & $9.90 \pm 6.30$ & 0.535 \\
HOP $(\mathrm{mg} / \mathrm{g})$ & $7.11 \pm 1.65$ & $7.18 \pm 1.74$ & 0.928 \\
\hline
\end{tabular}

\begin{tabular}{lccc}
\hline Variables & $\begin{array}{c}\text { SHR-C } \\
(\mathrm{n}=8)\end{array}$ & $\begin{array}{c}\text { SHR-SPR } \\
(\mathrm{n}=7)\end{array}$ & P value \\
\hline$\alpha$-MHC & $2.22 \pm 1.85$ & $3.77 \pm 1.57$ & 0.108 \\
$\beta$-MHC & $1.77 \pm 1.25$ & $2.34 \pm 0.77$ & 0.311 \\
ANP & $1.32 \pm 0.77$ & $1.15 \pm 0.47$ & 0.627 \\
Serca2a & $1.52 \pm 0.80$ & $1.83 \pm 0.52$ & 0.385 \\
\hline
\end{tabular}

Table 7. Left ventricular morphometric parameters and hydroxyproline concentration. Data are expressed as mean \pm standard deviation. SHR-C: spontaneously hypertensive rats without treatment; SHR-SPR: spontaneously hypertensive rats treated with spironolactone. CSA: myocyte cross-sectional area; ICF: interstitial collagen fractional area; HOP:

Table 8. Gene expression. Data are expressed as mean \pm standard deviation (arbitrary units). SHR-C: spontaneously hypertensive rats without treatment; SHR-SPR: spontaneously hypertensive rats treated with spironolactone. MHC: myosin heavy chain; ANP: atrial natriuretic peptide; Serca2a: sarcoplasmic reticulum calcium ATPase. Unpaired Student's $t$ test hydroxyproline. Unpaired Student's $t$ test

$2.25 \pm 0.19$ ) did not differ between groups. Gene expression for $\alpha$ - and $\beta$-myosin heavy chain, ANP, and Serca 2a was not different between groups (Table 8). Serca 2 and phospholamban protein levels were not statistically different between groups (Serca 2: SHR-C $1.00 \pm 0.36$; SHRSPR $0.93 \pm 0.27$ arbitrary units; $p=0.68$; phospholamban: SHR-C $1.00 \pm 0.51$; SHR-SPR $1.27 \pm$ 0.19 arbitrary units; $\mathrm{p}=0.21$ ). 


\section{Discussion}

In this study we showed that chronic administration of aldosterone blocker spironolactone to spontaneously hypertensive rats without the clinical features of heart failure reduces mortality without changing cardiac remodeling.

To the best of our knowledge, this is the first study to evaluate the long-term effects of early spironolactone treatment on SHR mortality, cardiac remodeling, and myocardial function. The spontaneously hypertensive rat is a well-established model of genetic hypertension $[1,2,26]$. At one month old, SHR begin to develop arterial hypertension and left ventricular hypertrophy, which often maintains cardiac performance despite the elevated systemic arterial pressure [44]. However, if pressure overload is sustained, cardiac decompensation may ensue, usually beginning at 18-22 months of age. After developing heart failure, rats evolve to death usually within two to four weeks $[1,2]$. In this study we evaluated the effects of aldosterone blocker during the transition from left ventricular hypertrophy to heart failure. Therefore, spironolactone was started when rats were 16 months old with no evidence of heart failure, and evaluated survival and ventricular and myocardial function six months later. Spironolactone has long been used to treat water and sodium retention. However, only after the study by Pitt et al. [21] has this drug attracted substantial attention from cardiologists and cardiovascular physiologists due to its potential to reduce the risk of morbidity and death in heart failure patients.

In this study, spironolactone did not change arterial systolic blood pressure. This result is in agreement with previous studies in SHR using similar spironolactone doses [45, 46]. As arterial pressure was unchanged by the treatment, we were able to exclude the influence of hemodynamic effects on the cardiac remodeling process and mortality. Untreated and treated groups presented a decrease in body weight during the experimental period. Body weight loss accompanying heart failure is an important predictor of mortality [47]. In our study, the body weight loss illustrates the advanced degree of heart failure.

Cardiac structures and left ventricular functional parameters evaluated by transthoracic echocardiogram did not differ between groups. Also, myocardial function, contractile reserve, and responsiveness to $\beta$-adrenergic stimulation assessed in papillary muscle preparations were not different between groups. Although not statistically significant, developed tension progressively decreased with increased isoproterenol doses in both groups (Table 5). Blunted responsiveness to $\beta$-adrenergic stimulation occurs early during myocardial hypertrophy and failure [48]. In SHR with long-standing hypertrophy, isoproterenol decreased papillary muscle isometric tension at Lmax [48]. Our study showed, therefore, that spironolactone does not modulate myocardial responsiveness to $\beta$-adrenergic stimulation.

Myocardial collagen content assessed by both biochemical and histological analyses was not different between groups, which is in accordance with unchanged myocardial and LV diastolic function. Cardiac remodeling is usually associated with changed gene expression from several myocardial proteins such as $\alpha$ - and $\beta$-myosin heavy chain, atrial natriuretic peptide, and Serca 2a. In our study, spironolactone treatment did not change expression of fetal genes program. Also, levels of Serca 2 and phospholamban, the proteins involved in intracellular calcium transient and myocardial contractility did not differ between groups. The results from gene and protein expression are in agreement with the lack of effects seen in cardiac structures and function.

In the clinical multicentric trials Rales [21], Ephesus [22], and Emphasis-HF [23], cardiac structural and functional evaluation was not performed after treatment with aldosterone blockers. Smaller clinical studies have shown conflicting results. In patients with mild-tomoderate systolic heart failure, some authors have observed aldosterone blockers-induced beneficial effects on structural and functional echocardiographic parameters [49-51], whereas others have found unchanged LV remodeling [52].

The effects of aldosterone blockers have been evaluated in different experimental models. In infarcted rodents, they have improved cardiac remodeling [14, 15, 19, 53, 54], whereas in dogs with rapid ventricular pacing-induced heart failure, eplerenone failed to prevent $\mathrm{LV}$ 
changes [55]. In SHR, the aldosterone blockade was evaluated during the cardiac remodeling process but not during heart failure development. In young [45, 56-59] and mature $[20,46]$ SHR, treatment with aldosterone blockers have resulted in improved or unchanged LV hypertrophy and myocardial fibrosis. The conflicting results after aldosterone blockers treatment suggest that their role in the remodeling process and heart failure development are not completely understood but are probably influenced by treatment period and experimental cardiac injury model.

Despite unchanged ventricular and myocardial remodeling and heart failure development, mortality rate was decreased in the spironolactone treated SHR. In the RALES [21] and EPHESUS [22] clinical trials, aldosterone blockers not only decreased cardiovascular and general mortality, but also sudden death. In patients with functional class III heart failure, spironolactone reduced ventricular extrasystole and non-sustained ventricular tachycardia [60]. Decreased urinary magnesium and potassium excretion has been suggested to play a role in lower ventricular arrhythmia rates [61]. However, in our study serum electrolytes concentration remained unchanged after treatment. In heart failure patients, spironolactone increased RR variability and reduced heart rate, QT interval, and QT dispersion, suggesting that it can increase parasympathetic tonus and have anti-arrhythmic properties [62].

During cardiac hypertrophy and heart failure, increased aldosterone production can induce electrical remodeling and cardiac arrhythmia by changing ion channels density or function [4, 63-69]. In vivo studies have shown that aldosterone blockers attenuate ventricular electrical remodeling and tachyarrhythmia vulnerability in different models of cardiac injury $[19,55$, 70]. Our results suggest that aldosterone blockade reduced mortality by decreasing cardiac arrhythmia and sudden death. We cannot refute, however, that cardiac remodeling was not changed because cardiac and myocardial evaluation was performed late after all non-treated rats with worse mechanical performance had died. Therefore, it is possible that by evaluating cardiac function and structures earlier, improved spironolactone-induced cardiac remodeling can be shown in spontaneous hypertensive rats.

In conclusion, early spironolactone administration reduces mortality without changing cardiac remodeling in spontaneous hypertensive rats.

\section{Conflict of Interest}

No conflict of interest declared

\section{Acknowledgements}

We are grateful to Jose Carlos Georgette and Elenize Jamas Pereira for their technical assistance and Colin Edward Knaggs for English editing. Financial support was provided by CNPq (305013/2009-0 and 304998/2009-5) and FAPESP (2007/57497-3, 2009/54407-9, and 2009/54506-7).

\section{References}

1 Bing OHL, Brooks WW, Robinson KG, Slawsky MT, Hayes JA, Litwin SE, Sen S, Conrad CH: The spontaneously hypertensive rat as a model of the transition from compensated left ventricular hypertrophy to failure. J Mol Cell Cardiol 1995;27:383-396.

2 Cicogna AC, Robinson KG, Conrad CH, Singh K, Squire R, Okoshi MP, Bing OHL: Direct effects of colchicine on myocardial function. Studies in hypertrophied and failing spontaneously hypertensive rats. Hypertension 1999;33:60-65. 


\section{Cellular Physiology $\quad$ Cell Physiol Biochem 2013;32:1275-1287

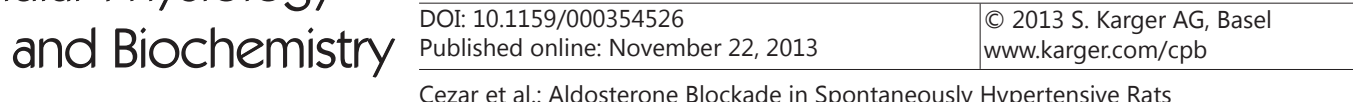

3 Messaoudi S, Azibani F, Delcayre C, Jaisser F: Aldosterone, mineralocorticoid receptor, and heart failure. Mol Cell Endocrinol 2012;350:266-272.

4 Mihailidou AS: Aldosterone in heart disease. Curr Hypertens Rep 2012;14:125-129.

5 Brilla CG, Matsubara LS, Weber KT: Anti-aldosterone treatment and the prevention of myocardial fibrosis in primary and secondary hyperaldosteronism. J Mol Cell Cardiol 1993;25:563-575.

6 Cheema Y, Zhao W, Zhao T, Khan MU, Green KD, Ahokas RA, Gerling IC, Bhattacharya SK, Weber KT: Reverse remodeling and recovery from cachexia in rats with aldosteronism. Am J Physiol Heart Circ Physiol 2012;303:H486-495.

7 Dartsch T, Fischer R, Gapelyuk A, Weiergraeber M, Ladage D, Schneider T, Schirdewan A, Reuter H, MuellerEhmsen J, Zobel C: Aldosterone induces electrical remodeling independent of hypertension. Int J Cardiol 2013;164:170-178.

-8 Mano A, Tatsumi T, Shiraishi J, Keira N, Nomura T, Takeda M, Nishikawa S, Yamanaka S, Matoba S, Kobara M, Tanaka H, Shirayama T, Takamatsu T, Nozawa Y, Matsubara H: Aldosterone directly induces myocyte apoptosis through calcineurin-dependent pathways. Circulation 2004;110:317-323.

-9 Okoshi MP, Yan X, Okoshi K, Nakayama M, Schuldt AJT, O'Connell T, Simpson PC, Lorell BH: Aldosterone directly stimulates cardiac myocyte hypertrophy. J Card Fail 2004;10:511-518.

10 Struthers AD, MacDonald TM: Review of aldosterone- and angiotensin II-induced target organ damage and prevention. Cardiovasc Res 2004;61:663-670.

11 Young MJ, Rickard AJ: Mechanisms of mineralocorticoid salt-induced hypertension and cardiac fibrosis. Mol Cell Endocrinol 2012;350:248-255.

12 Azibani F, Devaux Y, Coutance G, Schlossarek S, Polidano E, Fazal L, Merval R, Carrier L, Solal AC, Chatziantoniou C, Launay JM, Samuel JL, Delcayre C: Aldosterone inhibits the fetal program and increases hypertrophy in the heart of hypertensive mice. PLoS ONE 2012;7:e38197.

13 Enomoto S, Yoshiyama M, Omura T, Matsumoto R, Kusuyama T, Kim S, Izumi Y, Akioka K, Iwao H, Takeuchi $\mathrm{K}$, Yoshikawa J: Effects of eplerenone on transcriptional factors and mRNA expression related to cardiac remodelling after myocardial infarction. Heart 2005;91:1595-1600.

14 Fraccarollo D, Galuppo P, Schraut S, Kneitz S, Rooijen N, Ertl G, Bauersachs J: Immediate mineralocorticoid receptor blockade improves myocardial infarct healing by modulation of the inflammatory response. Hypertension 2008;51:905-914.

15 Katada J, Meguro T, Saito H, Ohashi A, Anzai T, Ogawa S, Yoshikawa T: Persistent cardiac aldosterone synthesis in angiotensin II type 1A receptor-knockout mice after myocardial infarction. Circulation 2005;111:2157-2164.

16 Kobayashi N, Yoshida K, Nakano S, Ohno T, Honda T, Tsubokou Y, Matsuoka H: Cardioprotective mechanisms of eplerenone on cardiac performance and remodeling in failing rat hearts. Hypertension 2006;47:671-679.

17 Kuster GM, Kotlyar E, Rude MK, Siwik DA, Liao R, Colucci WS, Sam F: Mineralocorticoid receptor inhibition ameliorates the transition to myocardial failure and decreases oxidative stress and inflammation in mice with chronic pressure overload. Circulation 2005;111:420-427.

18 Martin-Fernandez B, de Las Heras N, Miana M, Ballesteros S, Valero-Munoz M, Vassallo D, Davel AP, Rossoni LV, Cachofeiro V, Lahera V: Spironolactone prevents alterations associated with cardiac hypertrophy produced by isoproterenol in rats: involvement of SGK-1. Exp Physiol 2012;97:710-718.

19 Milliez P, Gomes S, Champ-Rigot L, Callebert J, Samuel JL, Delcayre C: Effects of spironolactone alone and in addition to a beta-blocker on myocardial histological and electrical remodeling in chronic severe failing rat hearts. J Cardiovasc Pharmacol 2012;60:315-321.

-20 Veliotes DG, Norton GR, Correia RJ, Strijdom H, Badenhorst D, Brooksbank R, Woodiwiss AJ: Impact of aldosterone receptor blockade on the deleterious cardiac effects of adrenergic activation in hypertensive rats. J Cardiovasc Pharmacol 2010;56:203-211.

21 Pitt B, Zannad F, Remme WJ, Cody R, Castaigne A, Perez A, Palensky J, Wittes J: The effect of spironolactone on morbidity and mortality in patients with severe heart failure. Randomized Aldactone Evaluation Study (RALES) Investigators. N Engl J Med 1999;341:709-717.

22 Pitt B, Remme W, Zannad F, Neaton J, Martinez F, Roniker B, Bittman R, Hurley S, Kleiman J, Gatlin M: Eplerenone, a selective aldosterone blocker, in patients with left ventricular dysfunction after myocardial infarction. N Engl J Med 2003;348:1309-1321.

23 Zannad F, McMurray JV, Krum H, van Veldhuisen DJ, Swedberg K, Shi H, Vincent J, Pocock SJ, Pitt B: Eplerenone in patients with systolic heart failure and mild symptoms. EMPHASIS-HF study group. N Engl J Med 2011;364:11-21. 


\section{Cellular Physiology $\quad$ Cell Physiol Biochem 2013;32:1275-1287 and Biochemistry \\ Cezar et al.: Aldosterone Blockade in Spontaneously Hypertensive Rats}

-24 Edelmann F, Wachter R, Schmidt AG, Kraigher-Krainer E, Colantonio C, Kamke W, Duvinage A, Stahrenberg R, Durstewitz K, Löffler M, Düngen HD, Tschöpe C, Herrmann-Lingen C, Halle M, Hasenfuss G, Gelbrich G, Pieske B: Effect of spironolactone on diastolic function and exercise capacity in patients with heart failure with preserved ejection fraction: the Aldo-DHF randomized controlled trial. JAMA 2013;309:781-791.

-25 McMurray JJV, Adamopoulos S, Anker SD, Auricchio A, Bohm M, Dickstein K, Falk V, Filippatos G, Fonseca C, Gomez-Sanchez MA, Jaarsma T, Kober L, Lip GYH, Maggioni AP, Parkhomenko A, Pieske BM, Popescu BA, Ronnevik PK, Rutten FH, Schwitter J, Seferovic P, Stepinska J, Trindade PT, Voors AA, Zannad F, Zeiher A: ESC Guidelines for the diagnosis and treatment of acute and chronic heart failure 2012. Eur Heart J 2012;33:17871847.

-26 Damatto RL, Martinez PF, Lima AR, Cezar MD, Campos DH, Oliveira Junior SA, Guizoni DM, Bonomo C, Nakatani BT, Dal Pai Silva M, Carvalho RF, Okoshi K, Okoshi MP: Heart failure-induced skeletal myopathy in spontaneously hypertensive rats. Int J Cardiol 2013;167:698-703.

27 Legrice IJ, Pope AJ, Sands GB, Whalley GA, Doughty RN, Smaill BH: Progression of myocardial remodeling and mechanical dysfunction in the spontaneously hypertensive rat. Am J Physiol Heart Circ Physiol 2012;303:H1353-1365.

28 Martinez PF, Okoshi K, Zornoff LAM, Oliveira Jr SA, Campos DHS, Lima ARR, Damatto RL, Cezar MDM, Bonomo C, Guizoni DM, Padovani CR, Cicogna AC, Okoshi MP: Echocardiographic detection of congestive heart failure in postinfarction rats. J Appl Physiol 2011;111:543-551.

-29 Santos PP, Nogueira BF, Rafacho BP, Azevedo PS, Polegato BF, Chiuso-Minicucci F, Bonomo C, Roscani MG, Zorzella-Pezavento SF, Tanni SE, Pereira EJ, Okoshi MP, Paiva SA, Zornoff LA, Minicucci MF: Aldosterone is not involved in the ventricular remodeling process induced by tobacco smoke exposure. Cell Physiol Biochem 2012;30:1191-1201.

-30 Okoshi K, Fioretto JR, Okoshi MP, Cicogna AC, Aragon FF, Matsubara LS, Matsubara BB: Food restriction induces in vivo ventricular dysfunction in spontaneously hypertensive rats without impairment of in vitro myocardial contractility. Braz J Med Biol Res 2004;37:607-613.

-31 Okoshi K, Ribeiro HB, Okoshi MP, Matsubara BB, Goncalves G, Barros R, Cicogna AC: Improved systolic ventricular function with normal myocardial mechanics in compensated cardiac hypertrophy. Jpn Heart J 2004;45:647-656.

-32 Paiva SAR, Zornoff LAM, Okoshi MP, Okoshi K, Matsubara LS, Matsubara BB, Cicogna AC, Campana AO: Ventricular remodeling induced by retinoic acid supplementation in adult rats. Am J Physiol Heart Circ Physiol 2003;284:H2242-2246.

-33 Lang RM, Bierig M, Devereux RB, Flachskampf FA, Foster E, Pellikka PA, Picard MH, Roman MJ, Seward J, Shanewise JS, Solomon SD, Spencer KT, Sutton MSJ, Stewart WJ: Recommendations for chamber quantification: a report from the American Society of Echocardiography's Guidelines and Standards Committee and the Chamber Quantification Writing Group, developed in conjunction with the European Association of Echocardiography, a branch of the European Society of Cardiology. J Am Soc Echocardiogr 2005;18:1440-1463.

-34 Litwin SE, Katz SE, Weinberg EO, Lorell BH, Aurigemma GP, Douglas PS: Serial echocardiographic-doppler assessment of left ventricular geometry and function in rats with pressure-overload hypertrophy. Chronic angiotensin-converting enzyme inhibition attenuates the transition to heart failure. Circulation 1995;91:26422654.

35 Cicogna AC, Padovani CR, Okoshi K, Matsubara LS, Aragon FF, Okoshi MP: The influence of temporal food restriction on the performance of isolated cardiac muscle. Nutr Res 2001;21:639-648.

-36 Okoshi MP, Okoshi K, Dal Pai V, Dal Pai-Silva M, Matsubara LS, Cicogna AC: Mechanical, biochemical, and morphological changes in the heart from chronic food restricted rats. Can J Physiol Pharmacol 2001;79:754760 .

-37 Sugizaki MM, Carvalho RF, Aragon FF, Padovani CR, Okoshi K, Okoshi MP, Zanati SG, Pai-Silva MD, Novelli EL, Cicogna AC: Myocardial dysfunction induced by food restriction is related to morphological damage in normotensive middle-aged rats. J Biomed Sci 2005;12:641-649.

-38 Okoshi MP, Matsubara LS, Franco M, Cicogna AC, Matsubara BB: Myocyte necrosis is the basis for fibrosis in renovascular hypertensive rats. Braz J Med Biol Res 1997;30:1135-1144.

-39 Fioretto JR, Querioz SS, Padovani CR, Matsubara LS, Okoshi K, Matsubara BB: Ventricular remodeling and diastolic myocardial dysfunction in rats submitted to protein-calorie malnutrition. Am J Physiol Heart Circ Physiol 2002;282:H1327-H1333. 


\section{Cellular Physiology $\quad$ Cell Physiol Biochem 2013;32:1275-1287 and Biochemistry \\ Cezar et al.: Aldosterone Blockade in Spontaneously Hypertensive Rats}

40 Lima ARR, Martinez PF, Okoshi K, Guizoni DM, Zornoff LAM, Campos DHS, Oliveira Jr SA, Bonomo C, Dal PaiSilva M, Okoshi MP: Myostatin and follistatin expression in skeletal muscles of rats with chronic heart failure. Int J Exp Path 2010;91:54-62.

41 Martinez PF, Okoshi K, Zornoff LAM, Carvalho RF, Oliveira Jr SA, Lima ARR, Campos DHS, Damatto RL, Nogueira CR, Dal Pai-Silva M, Okoshi MP: Chronic heart failure-induced skeletal muscle atrophy, necrosis, and myogenic regulatory factors changes. Med Sci Monit 2010;16:374-383.

-42 Parodi-Rullan R, Barreto-Torres G, Ruiz L, Casasnovas J, Javadov S: Direct renin inhibition exerts an antihypertrophic effect associated with improved mitochondrial function in post-infarction heart failure in diabetic rats. Cell Physiol Biochem 2012;29:841-850.

-43 Yan X, Schuldt AJ, Price RL, Amende I, Liu FF, Okoshi K, Ho KK, Pope AJ, Borg TK, Lorell BH, Morgan JP: Pressure overload-induced hypertrophy in transgenic mice selectively overexpressing AT2 receptors in ventricular myocytes. Am J Physiol Heart Circ Physiol 2008;294:H1274-1281.

44 Cicogna AC, Padovani CR, Okoshi K, Aragon FF, Okoshi MP: Myocardial function during chronic food restriction in isolated hypertrophied cardiac muscle. Am J Med Sci 2000;320:244-248.

45 Baldo MP, Forechi L, Morra EA, Zaniqueli D, Machado RC, Lunz W, Rodrigues SL, Mill JG: Long-term use of low-dose spironolactone in spontaneously hypertensive rats: effects on the left ventricular hypertrophy and stiffness. Pharmacol Rep 2011;63:975-982.

46 Kambara A, Holycross BJ, Wung P, Schanbacher B, Ghosh S, McCune SA, Bauer JA, Kwiatkowski P: Combined effectsof low-dose oral spironolactone and captopril therapy in a rat model of spontaneous hypertension and heart failure. J Cardiovasc Pharmacol 2003;41:830-837.

-47 Anker SD, Ponikowski P, Varney S, Chua TP, Clark AL, Webb-Peploe KM, Harrington D, Kox WJ, Poole-Wilson PA, Coats AJS: Wasting as independent risk factor of mortality in chronic heart failure. Lancet 1997;349:10501053.

-48 Brooks WW, Bing OH, Boluyt MO, Malhotra A, Morgan JP, Satoh N, Colucci WS, Conrad CH: Altered inotropic responsiveness and gene expression of hypertrophied myocardium with captopril. Hypertension 2000;35:1203-1209.

49 Boccanelli A, Mureddu GF, Cacciatore G, Clemenza F, Di Lenarda A, Gavazzi A, Porcu M, Latini R, Lucci D, Maggioni AP, Masson S, Vanasia M, de Simone G: Anti-remodelling effect of canrenone in patients with mild chronic heart failure (AREA IN-CHF study): final results. Eur J Heart Fail 2009;11:68-76.

50 Chan AKY, Sanderson JE, Wang T, Lam W, Yip G, Wang M, Lam Y-Y, Zhang Y, Yeung L, Wu EB, Chan WWM, Wong JTH, So N, Yu C-M: Aldosterone receptor antagonism induces reverse remodeling when added to angiotensin receptor blockade in chronic heart failure. J Am Coll Cardiol 2007;50:591-596.

51 Vizzardi E, D'Aloia A, Giubbini R, Bordonali T, Bugatti S, Pezzali N, Romeo A, Dei Cas A, Metra M, Dei Cas L: Effect of spironolactone on left ventricular ejection fraction and volumes in patients with class I or II heart failure. Am J Cardiol 2010;106:1292-1296.

52 Udelson JE, Feldman A, Greenberg B, Pitt B, Mukherjee R, Solomon HA, Konstam MA: Randomized, doubleblind, multicenter, placebo-controlled study evaluating the effect of aldosterone antagonism with eplerenone on ventricular remodeling in patients with mild-to-moderate heart failure and left ventricular systolic dysfunction. Circ Heart Fail 2010;3:347-353.

-53 Loan TY, Mardini M, Howell VM, Funder JW, Ashton AW, Mihailidou AS: Low-dose spironolactone prevents apoptosis repressor with caspase recruitment domain degradation during myocardial infarction. Hypertension 2012;59:1164-1169.

54 Masson S, Staszewsky L, Annoni G, Carlo E, Arosio B, Bai A, Calabresi C, Martinoli E, Salio M, Fiordaliso F, Scanziani E, Rudolph AE, Latini R: Eplerenone, a selective aldosterone blocker, improves diastolic function in aged rats with small-to-moderate myocardial infarction. J Card Fail 2004;10:433-441.

55 Stambler BS, Laurita KR, Shroff SC, Hoeker G, Martovitz NL: Aldosterone blockade attenuates development of an electrophysiological substrate associated with ventricular tachyarrhythmias in heart failure. Heart Rhythm 2009;6:776-783.

56 Burla AK, Neves MF, Oigman W, Mandarim-de-Lacerda CA: Eplerenone offsets cardiac and aortic adverse remodeling in spontaneously hypertensive rats. Int J Cardiol 2007;114:64-70.

57 Lacerda CAM, Pereira LMM: The effects of spironolactone monotherapy on blood pressure and myocardial remodeling in spontaneously hypertensive rats. A stereological study. J Biomed Sci 2003;10:50-57. 


\section{Cellular Physiology $\quad$ Cell Physiol Biochem 2013;32:1275-1287 and Biochemistry \\ Cezar et al.: Aldosterone Blockade in Spontaneously Hypertensive Rats}

58 Simko F, Pechanova O, Pelouch V, Krajcirovicova K, Mullerova M, Bednarova K, Adamcova M, Paulis L: Effect of melatonin, captopril, spironolactone and simvastatin on blood pressure and left ventricular remodelling in spontaneously hypertensive rats. J Hypertens 2009;27:5-10.

-59 Susic D, Varagic J, Frohlich ED: Cardiovascular effects of inhibition of renin-angiotensin-aldosterone system components in hypertensive rats given salt excess. Am J Physiol Heart Circ Physiol 2010;298:H1177-1181.

-60 Ramires FJ, Mansur A, Coelho O, Maranhao M, Gruppi CJ, Mady C, Ramires JA: Effect of spironolactone on ventricular arrhythmias in congestive heart failure secondary to idiopathic dilated or to ischemic cardiomyopathy. Am J Cardiol 2000;85:1207-1211.

61 Barr CS, Lang CC, Hanson J, Arnott M, Kennedy N, Struthers AD: Effects of adding spironolactone to an angiotensin-converting enzyme inhibitor in chronic congestive heart failure secondary to coronary artery disease. Am J Cardiol 1995;76:1259-1265.

62 Yee KM, Pringle SD, Struthers AD: Circadian variation in the effects of aldosterone blockade on heart rate variability and QT dispersion in congestive heart failure. J Am Coll Cardiol 2001;37:1800-1807.

63 Benitah JP, Perrier E, Gomez AM, Vassort G: Effects of aldosterone on transient outward $\mathrm{K}^{+}$current density in rat ventricular myocytes. J Physiol 2001;537:151-160.

64 Benitah JP, Vassort G: Aldosterone upregulates $\mathrm{Ca}^{2+}$ current in adult rat cardiomyocytes. Circ Res 1999;85:11391145.

65 Boixel C, Gavillet B, Rougier JS, Abriel H: Aldosterone increases voltage-gated sodium current in ventricular myocytes. Am J Physiol Heart Circ Physiol 2006;290:H2257-2266.

66 Cerbai E, Barbieri M, Mugelli A: Occurrence and properties of the hyperpolarization-activated current If in ventricular myocytes from normotensive and hypertensive rats during aging. Circulation 1996;94:1674-1681.

-67 Lalevee N, Rebsamen MC, Barrere-Lemaire S, Perrier E, Nargeot J, Benitah JP, Rossier MF: Aldosterone increases T-type calcium channel expression and in vitro beating frequency in neonatal rat cardiomyocytes. Cardiovasc Res 2005;67:216-224.

68 Mihailidou AS, Bundgaard H, Mardini M, Hansen PS, Kieldsen K, Rasmussen HH: Hyperaldosteronemia in rabbits inhibits the cardiac sarcolemmal $\mathrm{Na}^{+}-\mathrm{K}^{+}$pump. Circ Res 2000;86:37-42.

69 Muto T, Ueda N, Opthof T, Ohkusa T, Nagata K, Suzuki S, Tsuji Y, Horiba M, Lee JK, Honjo H, Kamiya K, Kodama I, Yasui K: Aldosterone modulates If current through gene expression in cultured neonatal rat ventricular myocytes. Am J Physiol Heart Circ Physiol 2007;293:H2710-2718.

70 Lammers C, Dartsch T, Brandt MC, Rottländer D, Halbach M, Peinkofer G, Ockenpoehler S, Weiergraeber M, Schneider T, Reuter H, Müller-Ehmsen J, Hescheler J, Hoppe UC, Zobel C: Spironolactone prevents aldosterone induced increased duration of atrial fibrillation in rat. Cell Physiol Biochem 2012;29:833-840. 